\title{
Numerical Solution of an Optimal Control Problem Governed by Two Dimensional Schrodinger Equation
}

\author{
Fatma Toyoglu, ", Gabil Yagubov ${ }^{1,2}$ \\ ${ }^{1}$ Department of Mathematics, Faculty of Art and Science, Erzincan University, Erzincan, Turkey \\ ${ }^{2}$ Department of Mathematics, Faculty of Art and Science, Kafkas University, Kars, Turkey
}

Email address:

ftoyoglu@erzincan.edu.tr (F. Toyoglu), gabilya@mail.ru (G. Yagubov)

\section{To cite this article:}

Fatma Toyoglu, Gabil Yagubov. Numerical Solution of an Optimal Control Problem Governed by Two Dimensional Schrodinger Equation. Applied and Computational Mathematics. Vol. 4, No. 2, 2015, pp. 30-38. doi: 10.11648/j.acm.20150402.11

\begin{abstract}
In this study, the finite difference method is applied to an optimal control problem controlled by two functions which are in the coefficients of two-dimensional Schrodinger equation. Convergence of the finite difference approximation according to the functional is proved. We have used the implicit method for solving the two-dimensional Schrodinger equation. Although the implicit scheme obtained from solution of the system of the linear equations is generally numerically stable and convergent without time-step condition, the solution of considered equation is numerically stable with time-step condition, due to the gradient term.
\end{abstract}

Keywords: Optimal Control, Schrodinger Operator, Finite Difference Methods, Stability, Convergence of Numerical Methods

\section{Introduction and Statement of Problem}

We consider the following optimal control problem:

$$
J(v)=\int_{0}^{l_{1}} \int_{0}^{2}\left|\psi\left(x_{1}, x_{2}, T\right)-y\left(x_{1}, x_{2}\right)\right|^{2} d x_{2} d x_{1} \rightarrow \min
$$

on the set

$$
V=\left\{v=v(t)=\left(v_{1}(t), v_{2}(t)\right), v_{p} \in L_{2}(0, T), p=1,2,\left\|v_{p}\right\|_{L_{2}(0, T)} \leq b_{p}, p=1,2\right\}
$$

subject to the problem;

$$
\begin{gathered}
i \frac{\partial \psi}{\partial t}+a_{0}\left(\frac{\partial^{2} \psi}{\partial x_{1}^{2}}+\frac{\partial^{2} \psi}{\partial x_{2}^{2}}\right)+i \sum_{j=1}^{2} a_{j}\left(x_{1}, x_{2}\right) \frac{\partial \psi}{\partial x_{j}}+\sum_{j=1}^{2} b_{j}\left(x_{1}, x_{2}\right) v_{j}(t) \psi-a\left(x_{1}, x_{2}\right) \psi=f\left(x_{1}, x_{2}, t\right),\left(x_{1}, x_{2}, t\right) \in \Omega, \\
\psi\left(x_{1}, x_{2}, 0\right)=\varphi\left(x_{1}, x_{2}\right),\left(x_{1}, x_{2}\right) \in D \\
\psi\left(0, x_{2}, t\right)=\psi\left(l_{1}, x_{2}, t\right)=\psi\left(x_{1}, 0, t\right)=\psi\left(x_{1}, l_{2}, t\right)=0, t \in(0, T],
\end{gathered}
$$

where $i^{2}=-1 ; a_{0}>0, T>0$ and $b_{p}>0, p=1,2$ are given numbers. $x=\left(x_{1}, x_{2}\right) \in D=\left(0, l_{1}\right) \times\left(0, l_{2}\right) \subset R^{2} ; \Omega_{t}=D \times(0, t)$; $\Omega=\Omega_{T} ; \quad y \in W_{2}^{1}(D)$ is a given function; $a, a_{j}, b_{j} \in L_{\infty}(D), j=1,2$ are given functions satisfying the following conditions: 


$$
\begin{aligned}
& 0 \leq a(x) \leq \mu_{0},\left|\frac{\partial a(x)}{\partial x_{k}}\right| \leq \mu_{1},\left|\frac{\partial^{2} a(x)}{\partial x_{k} \partial x_{p}}\right| \leq \mu_{2}, k, p=1,2, \quad \stackrel{0}{\forall} x \in D, \mu_{0}, \mu_{1}, \mu_{2}=\text { const. }>0, \\
& 0 \leq a_{j}(x) \leq v_{0},\left|\frac{\partial a_{j}(x)}{\partial x_{k}}\right| \leq v_{1},\left|\frac{\partial^{2} a_{j}(x)}{\partial x_{k} \partial x_{p}}\right| \leq v_{2}, j, p, k=1, \stackrel{0}{\forall} x \in D, v_{0}, v_{1}, v_{2}=\text { const. }>0, \\
& 0 \leq b_{j}(x) \leq \eta_{0},\left|\frac{\partial b_{j}(x)}{\partial x_{k}}\right| \leq \eta_{1},\left|\frac{\partial^{2} b_{j}(x)}{\partial x_{k} \partial x_{p}}\right| \leq \eta_{2}, j, p, k=1,2, \stackrel{0}{\forall} x \in D, \eta_{0}, \eta_{1}, \eta_{2}=\text { const. }>0 .
\end{aligned}
$$

Moreover we assume that the function $a_{j}$ satisfy the following condition:

$$
a_{j}\left(0, x_{2}\right)=a_{j}\left(l_{1}, x_{2}\right)=a_{j}\left(x_{1}, 0\right)=a_{j}\left(x_{1}, l_{2}\right)=0, j=1,2 .
$$

$\varphi\left(x_{1}, x_{2}\right)$ and $f\left(x_{1}, x_{2}, t\right)$ are given functions satisfying the following conditions:

$$
\stackrel{0}{\stackrel{0}{W_{2}^{2}}(D), f \in \stackrel{0}{W}_{2}^{2,0}(\Omega) .}
$$

Definitions of the given function spaces above are such as given in [9].

The optimal control problems for the Schrödinger equation have been investigated by different authors. Yetişkin and Subaş1 have studied the problem of determining of the potential in the Schrodinger equation from the measured final data [1]. Yıldiz at all.have investigated the existence and uniqueness of the solution of the optimal control problem for non-stationary Schrödinger equation and given necessary and sufficient conditions for the solution [2]. Beauchard and Laurent have investigated the exact controllability for the system which contain the linear Schrodinger equation, on a bounded interval, with a bilinear control in any positive time, locally around the ground state [3]. Yıldız and Subaşı have proved two estimates the solution of the optimal control problem for the linear Schrödinger equation and obtained necessary and sufficient conditions for the optimal solution [4] Baudouin at all.have obtained that the problem $i \partial_{t} u+\Delta u+|x-a(t)|^{-1} u+V_{1}(x, t) u=0 \quad$ with $u(x, 0)=u_{0}(x) \quad$ is well-posed and the regularity of the initial data is conserved for the solution if the electric potential $V_{1}$ is regular enough and at most quadratic at infinity [5].

Yagubov and Musayeva investigated finite-difference method solution of variation formulation of an inverse problem for nonlinear Schrodinger equation in [6].

This paper is organized as follows: In this section we state a

$$
\begin{gathered}
\left\{\left(x_{1 j_{1}}, x_{2 j_{2}}, t_{k}\right)_{n}\right\}, n=1,2, \ldots, x_{1 j_{1}}=j_{1} h_{1 n}-h_{1 n} / 2, j_{1}=\overline{1, M_{1 n}-1}, x_{2 j_{2}}=j_{2} h_{2 n}-h_{2 n} / 2, \\
j_{2}=\overline{1, M_{2 n}-1}, x_{11}-\frac{h_{1 n}}{2}=0, x_{1 M_{1 n}-1}+\frac{h_{1 n}}{2}=\ell_{1}, x_{21}-\frac{h_{2 n}}{2}=0, x_{2 M_{2 n}-1}+\frac{h_{2 n}}{2}=\ell_{2}, t_{k}=k \tau_{n}, \\
k=\overline{0, N_{n}}, h_{1}=h_{1 n}=\ell_{1} /\left(M_{1 n}-1\right), h_{2}=h_{2 n}=\ell_{2} /\left(M_{2 n}-1\right), \\
\tau=\tau_{n}=T / N_{n}, M_{1}=M_{1 n}, M_{2}=M_{2 n}, N=N_{n} .
\end{gathered}
$$

Finite differences for the space derivatives and the time derivatives in (2) are define in the following way according to $[7,8,10]$. theorem for the generalized solution of Schrödinger equation and then we discretize the given optimal control problem. In Section 2 we state and prove a theorem for the stability of the solution of finite difference approximations. In Section 3 we state a theorem for the error of the finite difference approximations. In the last section we state and prove two theorems for convergence of the finite difference approximations according to the functional.

Theorem 1: Suppose that the functions $a\left(x_{1}, x_{2}\right), a_{j}\left(x_{1}, x_{2}\right), b_{j}\left(x_{1}, x_{2}\right), j=1,2, \quad \varphi\left(x_{1}, x_{2}\right), f\left(x_{1}, x_{2}, t\right)$ hold the conditions (5)-(9). Then the initial-boundary value problem (2)-(4) has a unique solution belonging to the space $W_{2}^{2,1}(\Omega)$ for each $v \in V$ and this solution satisfies the following estimate:

$$
\|\psi\|_{W_{2}^{2,1}(\Omega)}^{2} \leq c_{0}\left(\|\varphi\|_{W_{2}^{2}(D)}^{2}+\|f\|_{W_{2}^{2,0}(\Omega)}^{2}\right)
$$

where the number $c_{0}>0$ is independent of $\varphi$ and $f$.

Proof of this problem can be obtained by similar processes given in [9].

Suppose that conditions (5)-(9) holds, then there exist the solution of the optimal control problem (1)-(4) according to [9].In other words, we can write the following:

$$
V_{*} \equiv\left\{v^{*} \in V: J\left(v^{*}\right)=J_{*}=\inf _{v \in V} J(v)\right\} \neq \varnothing .
$$

Now we solve the optimal control problem by using finite difference method. Firstly let us separate the domain $\bar{\Omega}=\left[0, l_{1}\right] \times\left[0, l_{2}\right] \times[0, T]$ using the meshes $0=x_{10}<x_{11} \ldots<x_{1 M_{1}}=l_{1}$ and $0=x_{20}<x_{21} \ldots<x_{2 M_{2}}=l_{2}$ in space and using a mesh $0=t_{0}<t_{1}<\ldots<t_{N}=T$ in time. Here the mesh points are following: 


$$
\begin{aligned}
& \delta_{\bar{t}} \phi_{j_{1} j_{2} k}=\left(\phi_{j_{1} j_{2} k}-\phi_{j_{1} j_{2} k-1}\right) / \tau \text {, } \\
& \delta_{\bar{x}_{1}} \phi_{j_{1} j_{2} k}=\left(\phi_{j_{1} j_{2} k}-\phi_{j_{1}-1 j_{2} k}\right) / h_{1}, \quad \delta_{\bar{x}_{2}} \phi_{j_{1} j_{2} k}=\left(\phi_{j_{1} j_{2} k}-\phi_{j_{1} j_{2}-1 k}\right) / h_{2}, \\
& \delta_{x_{1}} \phi_{j_{1} j_{2} k}=\left(\phi_{j_{1}+1 j_{2} k}-\phi_{j_{1} j_{2} k}\right) / h_{1}, \quad \delta_{x_{2}} \phi_{j_{1} j_{2} k}=\left(\phi_{j_{1} j_{2}+1 k}-\phi_{j_{1} j_{2} k}\right) / h_{2}, \\
& \delta_{x_{1} \bar{x}_{1}} \phi_{j_{1} j_{2} k}=\left(\phi_{j_{1}+1 j_{2} k}-2 \phi_{j_{1} j_{2} k}+\phi_{j_{1}-1 j_{2} k}\right) / h_{1}^{2}, \quad \delta_{x_{2} \bar{x}_{2}} \phi_{j_{j} j_{2} k}=\left(\phi_{j_{j} j_{2}+1 k}-2 \phi_{j_{1} j_{2} k}+\phi_{j_{1} j_{2}-1 k}\right) / h_{2}^{2} \text {, } \\
& \delta_{\bar{x}_{1}} \phi_{1 j_{2} k}=\delta_{x_{1}} \phi_{0 j_{2} k}=\frac{\left(\phi_{1 j_{2} k}-\phi_{0 j_{2} k}\right)}{\left(h_{1} / 2\right)}, \quad \delta_{\bar{x}_{1}} \phi_{M_{1} j_{2} k}=\delta_{x_{1}} \phi_{M_{1}-1 j_{2} k}=\frac{\left(\phi_{M_{1} j_{2} k}-\phi_{M_{1}-1 j_{2} k}\right)}{\left(h_{1} / 2\right)}, \\
& \delta_{\bar{x}_{2}} \phi_{j_{1} 1 k}=\delta_{x_{2}} \phi_{j_{1} 0 k}=\frac{\left(\phi_{j_{1} 1 k}-\phi_{j_{0} 0 k}\right)}{\left(h_{2} / 2\right)}, \quad \delta_{\bar{x}_{2}} \phi_{j_{1} M_{2} k}=\delta_{x_{2}} \phi_{j_{1} M_{2}-1 k}=\frac{\left(\phi_{j_{1} M_{2} k}-\phi_{j_{1} M_{2}-1 k}\right)}{\left(h_{2} / 2\right)} .
\end{aligned}
$$

Hence finite difference approximations are substituted for the derivatives to convert the optimal control problem (1)-(4) to an algebraic form for any integer $n \geq 1$ as following problem according to [10]:

$$
I_{n}\left([v]_{n}\right)=h_{1} h_{2} \sum_{j_{1}=1}^{M_{1}-1} \sum_{j_{2}=1}^{M_{2}-1}\left|\phi_{j_{1} j_{2} N}-y_{j_{1} j_{2}}\right|^{2} \rightarrow \min
$$

on the set

$$
\begin{gathered}
V_{n} \equiv\left\{[v]_{n}:[v]_{n}=\left(\left[v_{1}\right]_{n},\left[v_{2}\right]_{n}\right),\left[v_{p}\right]_{n}=\left(v_{p 1}, v_{p 2}, \ldots, v_{p N}\right), p=1,2,\right. \\
\left.\left(\tau \sum_{k=1}^{N}\left|v_{p k}\right|^{2}\right)^{1 / 2} \leq b_{p}, p=1,2, k=\overline{1, N}\right\}
\end{gathered}
$$

subject to the problem

$$
\begin{aligned}
& i \delta_{\bar{t}} \phi_{j_{1} j_{2} k}+a_{0}\left(\delta_{x_{1} \overline{x_{1}}} \phi_{j_{1} j_{2} k}+\delta_{x_{2} \bar{x}_{2}} \phi_{j_{1} j_{2} k}\right)+i \sum_{j=1}^{2} a_{j}^{j_{j} j_{2}} \delta_{\bar{x}_{j}} \phi_{j_{1} j_{2} k}+\sum_{j=1}^{2} b_{j}^{j_{j} j_{2}} v_{j k} \phi_{j_{1} j_{2} k}-a^{j_{1} j_{2}} \phi_{j_{1} j_{2} k}=f_{j_{1} j_{2} k}, \\
& j_{1}=\overline{1, M_{1}-1}, j_{2}=\overline{1, M_{2}-1}, k=\overline{1, N}, \\
& \phi_{j_{1} j_{2} 0}=\varphi_{j_{1} j_{2}}, j_{1}=\overline{0, M_{1}}, j_{2}=\overline{0, M_{2}}, \\
& \phi_{0 j_{2} k}=\phi_{M_{1} j_{2} k}=0, j_{2}=\overline{0, M_{2}}, k=\overline{1, N} \text {, } \\
& \phi_{j_{1} 0 k}=\phi_{j_{1} M_{2} k}=0, j_{1}=\overline{0, M_{1}}, k=\overline{1, N} . \\
& a_{j}^{j_{j} j_{2}}=\frac{1}{h_{1} h_{2}} \int_{x_{1 j}-h_{1} / 2}^{x_{i j_{1}}+h_{1} / 2} \int_{x_{2 / 2}-h_{2} / 2}^{x_{2 j}+h_{2} / 2} a_{j}\left(x_{1}, x_{2}\right) d x_{2} d x_{1}, j=1,2, j_{1}=\overline{1, M_{1}-1}, j_{2}=\overline{1, M_{2}-1}, \\
& b_{j}^{j_{j} j_{2}}=\frac{1}{h_{1} h_{2}} \int_{x_{j_{1}}-h_{h_{1}} / 2 x_{2 j_{2}}-h_{2} / 2}^{x_{i_{1}}+h_{1} / 2 x_{2 j_{2}}+h_{2} / 2} b_{j}\left(x_{1}, x_{2}\right) d x_{2} d x_{1}, j=1,2, j_{1}=\overline{1, M_{1}-1}, j_{2}=\overline{1, M_{2}-1}, \\
& a^{j_{1} j_{2}}=\frac{1}{h_{1} h_{2}} \int_{x_{1 j}-h_{1} / 2}^{x_{1 j}+h_{1} / 2} \int_{x_{2 / 2}-h_{2} / 2}^{x_{2 / 2}+h_{2} / 2} a\left(x_{1}, x_{2}\right) d x_{2} d x_{1}, j_{1}=\overline{1, M_{1}-1}, j_{2}=\overline{1, M_{2}-1}, \\
& \varphi_{j_{1}, j_{2}}=\frac{1}{h_{1} h_{2}} \int_{x_{1, j}-h_{1} / 2}^{x_{1 j_{1}}+h_{1} / 2 x_{2, j_{2}}-h_{2} / 2} \int_{x_{2}+h_{2} / 2} \varphi\left(x_{1}, x_{2}\right) d x_{2} d x_{1}, j_{1}=\overline{1, M_{1}-1}, j_{2}=\overline{1, M_{2}-1}, \\
& \varphi_{00}=\varphi_{0 M_{2}}=\varphi_{M_{1} 0}=\varphi_{M_{1} M_{2}}=0,
\end{aligned}
$$

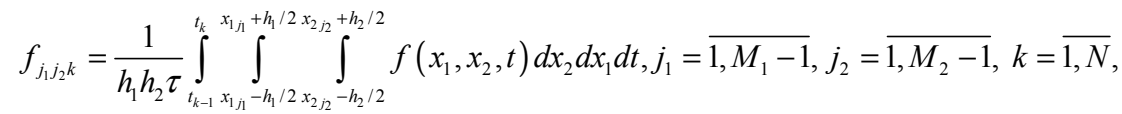




$$
y_{j_{1}, j_{2}}=\frac{1}{h_{1} h_{2}} \int_{x_{1, j}-h_{1} / 2}^{x_{1, j}+h_{1} / 2} \int_{x_{2 / 2}-h_{2} / 2} y\left(x_{1}, x_{2}\right) d x_{2} d x_{1}, j_{1}=\overline{1, M_{1}-1}, j_{2}=\overline{1, M_{2}-1} .
$$

Using the equalities (16)-(18) and the conditions on $a, a_{j}, b_{j}$ given with (5)-(8), we find

$$
\begin{aligned}
& 0 \leq a^{j_{1} j_{2}} \leq \mu_{0},\left|\delta_{\bar{x}_{k}} a^{j_{1} j_{2}}\right| \leq \mu_{1},\left|\delta_{x_{k} \bar{x}_{p}} a^{j_{1} j_{2}}\right| \leq \mu_{2}, k, p=1,2, \\
& 0 \leq a_{j}^{j_{1} j_{2}} \leq v_{0},\left|\delta_{\bar{x}_{k}} a_{j}^{j_{j} j_{2}}\right| \leq v_{1},\left|\delta_{x_{k_{k}} \bar{x}_{p}} a_{j}^{j_{j} j_{2}}\right| \leq v_{2}, j, k, p=1,2, \\
& a_{j}^{0 j_{2}}=a_{j}^{M_{1} j_{2}}=0, a_{j}^{j_{1} 0}=a_{j}^{j_{1} M_{2}}=0, \\
& 0 \leq b_{j}^{j_{1} j_{2}} \leq \eta_{0},\left|\delta_{\bar{x}_{k}} b_{j}^{j_{j} j_{2}}\right| \leq \eta_{1},\left|\delta_{x_{k} \overline{x_{p}}} b_{j}^{j_{1} j_{2}}\right| \leq \eta_{2}, j, k, p=1,2 .
\end{aligned}
$$

\section{Stability of the Finite Difference Approximations}

In this section, we examine whether the solution of finite difference approximations is stable or not.

Theorem 2: Suppose that the time-step $\tau$ satisfy the

$$
h_{1} h_{2} \sum_{j_{1}=1}^{M_{1}-1} \sum_{j_{2}=1}^{M_{2}-1}\left|\phi_{j_{1} j_{2} m}\right|^{2} \leq c_{1}\left(h_{1} h_{2} \sum_{j_{1}=1}^{M_{1}-1} \sum_{j_{2}=1}^{M_{2}-1}\left|\varphi_{j_{1}, j_{2}}\right|^{2}+h_{1} h_{2} \tau \sum_{k=1}^{N} \sum_{j_{1}=1}^{M_{1}-1} \sum_{j_{2}=1}^{M_{2}-1}\left|f_{j_{1} j_{2} k}\right|^{2}\right), m \in\{1,2, \ldots, N\}
$$

Here the number $c_{1}>0$ is independent of $h_{1}, h_{2}$ and $\tau$.

Proof: The scheme (12)-(15) is equivalent to the following identity for each $t=t_{k}$ :

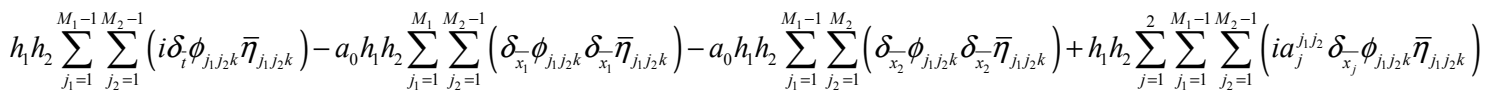

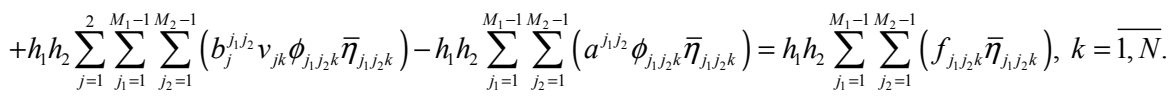

Here the mesh function $\bar{\eta}_{j_{1} j_{2} k}$ is complex conjugate of the arbitrary mesh function $\eta_{j_{1} j_{2} k}$ which is defined on the mesh sequence $\left\{\left(x_{1 j_{1}}, x_{2 j_{2}}, t_{k}\right)_{n}\right\}$ and satisfies the conditions $\eta_{0 j_{j k} k}=\eta_{M_{1} j_{2} k}=0, \eta_{j_{1} 0 k}=\eta_{j_{1} M_{2} k}=0, j_{1}=\overline{0, M_{1}}, j_{2}=\overline{0, M_{2}}, \mathrm{k}=\overline{1, N}$. Here substituting $\bar{\eta}_{j_{1} j_{2} k}$ with $\tau \bar{\phi}_{j_{1} j_{2} k}$ and then subtracting complex conjugate of obtained equality from itself, we have the following equation:

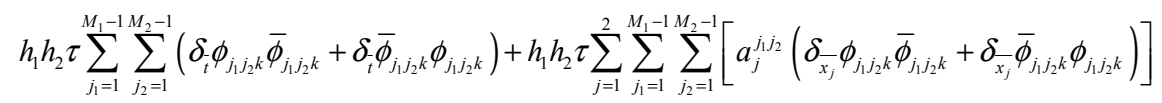

$$
\begin{aligned}
& =2 h_{1} h_{2} \tau \sum_{j_{1}=1}^{M_{1}-1} \sum_{j_{2}=1}^{M_{2}-1} \operatorname{Im}\left(f_{j, j_{j} k} \bar{\phi}_{j, j_{2} k}\right), \forall k \in\{1,2, \ldots, N\} \text {. }
\end{aligned}
$$

Using

$$
\begin{gathered}
\tau\left(\delta_{t} \phi_{j_{1} j_{2} k} \bar{\phi}_{j_{1} j_{2} k}+\delta_{i} \bar{\phi}_{j_{1} j_{2} k} \phi_{j_{1} j_{2} k}\right)=\left|\phi_{j_{1} j_{2} k}\right|^{2}-\left|\phi_{j_{1} j_{2} k-1}\right|^{2}+\left|\phi_{j_{1} j_{2} k}-\phi_{j_{1} j_{2} k-1}\right|^{2} \\
h_{1}\left(\delta_{x_{1}} \phi_{j_{1} j_{2}} \bar{\phi}_{j_{1} j_{2} k}+\delta_{\bar{x}_{1}} \bar{\phi}_{j_{1} j_{2} k} \phi_{j_{1} j_{2} k}\right)=\left|\phi_{j_{1} j_{2} k}\right|^{2}-\left|\phi_{j_{1}-1 j_{2} k}\right|^{2}+\left|\phi_{j_{1} j_{2} k}-\phi_{j_{1}-1 j_{2} k}\right|^{2} \\
h_{2}\left(\delta_{\bar{x}_{2}} \phi_{j_{1} j_{2} k} \bar{\phi}_{j_{1} j_{2} k}+\delta_{\bar{x}_{2}} \bar{\phi}_{j_{1} j_{2} k} \phi_{j_{1} j_{2} k}\right)=\left|\phi_{j_{1} j_{2} k}\right|^{2}-\left|\phi_{j_{1} j_{2}-1 k}\right|^{2}+\left|\phi_{j_{1} j_{2} k}-\phi_{j_{1} j_{2}-1 k}\right|^{2}
\end{gathered}
$$

in (27), adding index $k$ from 1 through $m \leq N$ and then calculating absolute value of both sides, and we use the condition (23), we have the following: 


$$
\begin{aligned}
& h_{1} h_{2} \sum_{j_{1}=1}^{M_{1}-1} \sum_{j_{2}=1}^{M_{2}-1}\left|\phi_{j_{j}, j_{2} m}\right|^{2} \leq h_{1} h_{2} \sum_{j_{1}=1}^{M_{1}-1} \sum_{j_{2}=1}^{M_{2}-1}\left|\varphi_{j_{1} j_{2}}\right|^{2}+h_{1} h_{2} \tau \sum_{k=1}^{m} \sum_{j_{1}=2}^{M_{1}} \sum_{j_{2}=1}^{M_{2}-1}\left|\delta_{\bar{T}_{1}} a_{1}^{j_{1}, j_{j}}\right|\left|\phi_{j_{1}-1 j_{2} k}\right|^{2} \\
& +h_{1} h_{2} \tau \sum_{k=1}^{m} \sum_{j_{1}=1}^{M_{1}-1} \sum_{j_{2}=2}^{M_{2}}\left|\delta_{\bar{x}_{2}} a_{2}^{j_{j} j_{2}}\right|\left|\phi_{j, j_{2}-1 k}\right|^{2}+2 h_{1} h_{2} \tau \sum_{k=1}^{m} \sum_{j_{1}=1}^{M_{1}-1} \sum_{j_{2}=1}^{M_{2}-1}\left|f_{j_{j}, j_{k} k}\right|\left|\phi_{j, j, k}\right| \\
& \leq h_{1} h_{2} \sum_{j_{1}=1}^{M_{1}-1} \sum_{j_{2}=1}^{M_{2}-1}\left|\varphi_{j_{1} j_{2}}\right|^{2}+2 v_{1} h_{1} h_{2} \tau \sum_{k=1}^{m} \sum_{j_{1}=1}^{M_{1}-1} \sum_{j_{2}=1}^{M_{2}-1}\left|\phi_{j_{j}, j_{2}}\right|^{2} \\
& +2 h_{1} h_{2} \tau \sum_{k=1}^{m} \sum_{j_{1}=1}^{M_{1}-1} \sum_{j_{2}=1}^{M_{2}-1}\left|f_{j_{j} j_{2} k}\right|\left|\phi_{j, j_{2} k}\right|, \forall m \in\{1,2, \ldots, N\}
\end{aligned}
$$

The $m t h$ term of the last term on the right side of this inequality writing a separate and then applying $\varepsilon-$ Cauchy and Cauchy-Bunyakovsky inequalities, we get the following inequality:

$$
\begin{aligned}
h_{1} h_{2} \sum_{j_{1}=1}^{M_{1}-1} \sum_{j_{2}=1}^{M_{2}-1}\left|\phi_{j_{j} j_{2} m}\right|^{2} & \leq h_{1} h_{2} \sum_{j_{1}=1}^{M_{1}-1} \sum_{j_{2}=1}^{M_{2}-1}\left|\varphi_{j_{1} j_{2}}\right|^{2}+2 v_{1} h_{1} h_{2} \tau \sum_{k=1}^{m} \sum_{j_{1}=1}^{m} \sum_{j_{2}=1}^{M_{1}-1}\left|\phi_{j_{j} j_{2} k}\right|^{2}+\varepsilon h_{1} h_{2} \tau \sum_{j_{1}=1}^{M_{1}-1} \sum_{j_{2}=1}^{M_{2}-1}\left|f_{j_{j} j_{2} m}\right|^{2}+\frac{1}{\varepsilon} h_{1} h_{2} \tau \sum_{j_{1}=1}^{M_{1}-1} \sum_{j_{2}=1}^{M_{2}-1}\left|\phi_{j_{j} j_{2} m}\right|^{2} \\
& +h_{1} h_{2} \tau \sum_{k=1}^{m-1} \sum_{j_{1}=1}^{M_{1}-1} \sum_{j_{2}=1}^{M_{2}-1}\left|f_{j_{1} j_{2} k}\right|^{2}+h_{1} h_{2} \tau \sum_{k=1}^{m-1} \sum_{j_{1}=1}^{M_{1}-1} \sum_{j_{2}=1}^{M_{2}-1}\left|\phi_{j_{j} j_{2} k}\right|^{2}, \forall m \in\{1,2, \ldots, N\}
\end{aligned}
$$

Writing $\varepsilon=2 \tau$, we obtain

$$
\begin{aligned}
& h_{1} h_{2} \sum_{j_{1}=1}^{M_{1}-1} \sum_{j_{2}=1}^{M_{2}-1}\left|\phi_{j_{1} j_{2} m}\right|^{2} \leq 2 h_{1} h_{2} \sum_{j_{1}=1}^{M_{1}-1} \sum_{j_{2}=1}^{M_{2}-1}\left|\varphi_{j_{1} j_{2}}\right|^{2}+(4 T+2) h_{1} h_{2} \tau \sum_{k=1}^{m} \sum_{j_{1}=1}^{M_{1}-1} \sum_{j_{2}=1}^{M_{2}-1}\left|f_{j_{j}, j_{2} k}\right|^{2} \\
& +4 v_{1} h_{1} h_{2} \tau \sum_{k=1}^{m} \sum_{j_{1}=1}^{M_{1}-1} \sum_{j_{2}=1}^{M_{2}-1}\left|\phi_{j_{1} j_{2} k}\right|^{2}+2 h_{1} h_{2} \tau \sum_{k=1}^{m-1} \sum_{j_{1}=1}^{M_{1}-1} \sum_{j_{2}=1}^{M_{2}-1}\left|\phi_{j_{1} j_{2}}\right|^{2}, m=\overline{1, N}
\end{aligned}
$$

and then the $m t h$ term of the third term on the right side writing a separate and using the condition $0<\tau \leq \frac{1}{8}\left(v_{1}\right)^{-1}$ for time-step $\tau$, we have the following:

$$
h_{1} h_{2} \sum_{j_{1}=1}^{M_{1}-1} \sum_{j_{2}=1}^{M_{2}-1}\left|\phi_{j_{j} j_{2} m}\right|^{2} \leq 4 h_{1} h_{2} \sum_{j_{1}=1}^{M_{1}-1} \sum_{j_{2}=1}^{M_{2}-1}\left|\varphi_{j_{j} j_{2}}\right|^{2}+(8 T+4) h_{1} h_{2} \tau \sum_{k=1}^{N} \sum_{j_{1}=1}^{M_{1}-1} \sum_{j_{2}=1}^{M_{2}-1}\left|f_{j_{1} j_{2} k}\right|^{2}+\left(8 v_{1}+4\right) h_{1} h_{2} \tau \sum_{k=0}^{m-1} \sum_{j_{1}=1}^{M_{1}-1} \sum_{j_{2}=1}^{M_{2}-1}\left|\phi_{j_{j} j_{2} k}\right|^{2}, \quad m \in\{1,2, \ldots, N\}
$$

Using Gronwall Lemma in this inequality, we get the following inequality:

$$
h_{1} h_{2} \sum_{j_{1}=1}^{M_{1}-1} \sum_{j_{2}=1}^{M_{2}-1}\left|\phi_{j_{j}, m}\right|^{2} \leq c_{2}\left(h_{1} h_{2} \sum_{j_{1}=1}^{M_{1}-1} \sum_{j_{2}=1}^{M_{2}-1}\left|\varphi_{j_{j} j_{2}}\right|^{2}+h_{1} h_{2} \tau \sum_{k=1}^{N} \sum_{j_{1}=1}^{M_{1}-1} \sum_{j_{2}=1}^{M_{2}-1}\left|f_{j_{j, j} k}\right|^{2}\right), m \in\{1,2, \ldots, N\}
$$

Here the number $c_{2}>0$ is independent of $h_{1}, h_{2}$ and $\tau$.

\section{An Estimate for the Error of the Finite Difference Approximations}

Firstly, let us define the solution $\psi=\psi(x, t ; v)$ of the optimal control problem (1)-(4) for each $v \in V$ such as following:

$$
\begin{aligned}
& {\left[\psi\left(x_{1}, x_{2}, t ; v\right)\right]_{n}=\left\{\psi_{j, j, j_{2} k}\right\},}
\end{aligned}
$$

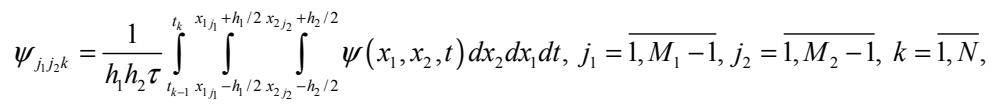

$$
\begin{aligned}
& \psi_{j, j_{2} 0}=\varphi_{j, j, 2}, j_{1}=\overline{0, M_{1}}, j_{2}=\overline{0, M_{2}}, \\
& \psi_{0 j_{2 k} k}=\psi_{M_{1, j k} k}=0, j_{2}=\overline{0, M_{2}}, k=\overline{1, N} \text {, } \\
& \psi_{j_{1} 0 k}=\psi_{j, M_{2} k}=0, j_{1}=\overline{0, M_{1}}, k=\overline{1, N} .
\end{aligned}
$$

Let us define the operator $Q_{n}$ on the set $V$ for each $v \in V$ such as following:

$$
\begin{gathered}
Q_{n}(v): V \rightarrow V_{n}, Q_{n}(v)=[w]_{n}=\left(\left[w_{1}\right]_{n},\left[w_{2}\right]_{n}\right), Q_{n}(v)=\left(w_{p 1}, w_{p 2}, \ldots, w_{p N}\right), p=1,2, \\
w_{p k}=\frac{1}{\tau} \int_{t_{k-1}}^{t_{k}} v_{p}(t) d t, p=1,2, k=\overline{1, N}
\end{gathered}
$$


$[Z]_{n}=\left\{Z_{j_{j} j_{2} k}\right\}=\left\{\phi_{j_{j} j_{2} k}-\psi_{j_{j} j_{2} k}\right\}$ is error of the finite difference approximations. The mesh function $\left\{Z_{j_{j, j_{2} k}}\right\}$ satisfies the following problem:

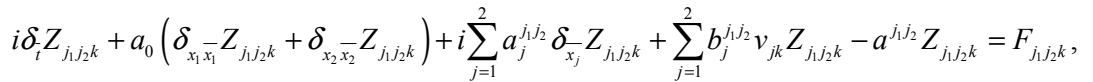

$$
\begin{aligned}
& j_{1}=\overline{1, M_{1}-1}, j_{2}=\overline{1, M_{2}-1}, k=\overline{1, N}, \\
& Z_{j_{1} j_{2} 0}=0, j_{1}=\overline{0, M_{1}}, j_{2}=\overline{0, M_{2}}, \\
& Z_{0 j_{2} k}=Z_{M_{1} j_{2} k}=0, j_{2}=\overline{0, M_{2}}, k=\overline{1, N}, Z_{j_{1} 0 k}=Z_{j_{1} M_{2} k}=0, j_{1}=\overline{0, M_{1}}, k=\overline{1, N} .
\end{aligned}
$$

Here the function $F_{j_{1} j_{2} k}$ is defined such as the following:

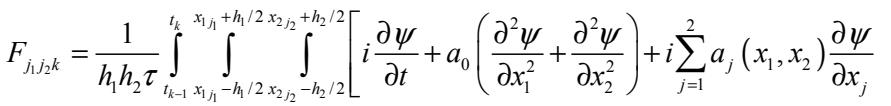

$$
\begin{aligned}
& \left.+\sum_{j=1}^{2} b_{j}\left(x_{1}, x_{2}\right) v_{j}(t) \psi-a\left(x_{1}, x_{2}\right) \psi\right] d x_{2} d x_{1} d t-i \delta_{i} \psi_{j, j_{2} k}-a_{0}\left(\delta_{x_{1} \bar{X}_{1}} \psi_{j, j_{2} k}+\delta_{x_{2} \bar{x}_{2}} \psi_{j_{i, j} k}\right) \\
& -i \sum_{j=1}^{2} a_{j}^{j_{j} j_{2}} \delta_{x_{j}} \psi_{j_{1} j_{2} k}-\sum_{j=1}^{2} b_{j}^{j_{j} j_{2}} v_{j k} \psi_{j_{1} j_{2} k}+a^{j_{1} j_{2}} \psi_{j_{1} j_{2} k}, \quad j_{1}=\overline{1, M_{1}-1}, j_{2}=\overline{1, M_{2}-1}, k=\overline{1, N} .
\end{aligned}
$$

Theorem 3: Suppose that the time-step $\tau$ satisfy the condition $0<\tau \leq \frac{1}{8}\left(v_{1}\right)^{-1}$ and space-steps $h_{1}, h_{2}$ satisfy the adaptation conditions $c_{3} \leq \frac{h_{1}}{\tau} \leq c_{4}$ and $c_{5} \leq \frac{h_{2}}{\tau} \leq c_{6}$. Here the numbers $c_{3}>0, c_{4}>0, c_{5}>0, c_{6}>0$ are independent of $\tau, h_{1}$ and $h_{2}$. Then the following estimate is valid:

$$
h_{1} h_{2} \sum_{j_{1}=1}^{M_{1}-1} \sum_{j_{2}=1}^{M_{2}-1}\left|Z_{j_{1} j_{2} m}\right|^{2} \leq c_{7}\left(\beta_{\tau h_{1} h_{2}}+\left\|Q_{n}(v)-[v]_{n}\right\|^{2}\right), \forall m \in\{1,2, \ldots, N\}
$$

Here the number $c_{7}>0$ is independent of $h_{1}, h_{2}$ and $\tau$. $\beta_{\tau h_{1} h_{2}}>0, \quad \beta_{\tau h_{1} h_{2}} \rightarrow 0$ for $\tau \rightarrow 0, h_{1} \rightarrow 0$ and $h_{2} \rightarrow 0$. Moreover the following equality is satisfied;

$$
\left\|Q_{n}(v)-[v]_{n}\right\|^{2}=\left(\tau \sum_{k=1}^{N} \sum_{p=1}^{2}\left|w_{p k}-v_{p k}\right|^{2}\right) .
$$

\section{Convergence of the Finite Difference Approximations According to the Functional}

In this section, we prove convergence of the finite difference approximations according to the functional. First,

$$
\begin{aligned}
& J(v)-I_{n}\left([v]_{n}\right)=\int_{0}^{h_{1}} \int_{0}^{l}\left|\psi\left(x_{1}, x_{2}, T\right)-y\left(x_{1}, x_{2}\right)\right|^{2} d x_{2} d x_{1}-h_{1} h_{2} \sum_{j_{1}=1}^{M_{1}-1} \sum_{j_{2}=1}^{M_{2}-1}\left|\phi_{j, j_{2} N}-y_{j, j_{2}}\right|^{2}
\end{aligned}
$$

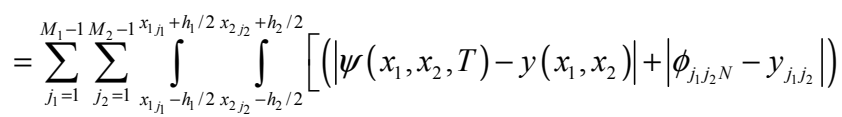

$$
\begin{aligned}
& \left.\times\left(\left|\psi\left(x_{1}, x_{2}, T\right)-y\left(x_{1}, x_{2}\right)\right|-\left|\phi_{j_{1} j_{2} N}-y_{j_{1} j_{2}}\right|\right)\right] d x_{2} d x_{1} .
\end{aligned}
$$

Applying Cauchy-Bunyakovsky inequality and using (10) and (25) in this equality, we have: let us estimate the finite difference between the functionals $J(v)$ and $I_{n}\left([v]_{n}\right)$.

Theorem 4: Suppose that the conditions of Theorem 3 are hold. Then the following inequality is valid for $\forall v \in V$ and $\forall[v]_{n} \in V_{n}:$

$$
\left|J(v)-I_{n}\left([v]_{n}\right)\right| \leq c_{8}\left(\sqrt{\beta_{h_{1} h_{2} \tau}}+\left\|Q_{n}(v)-[v]_{n}\right\|\right) .
$$

Here the number $c_{8}>0$ is independent $h_{1}, h_{2}$ and $\tau$.

Proof: We consider the difference $J(v)-I_{n}\left([v]_{n}\right)$. Using (1) and (11) we can write the following equality: 


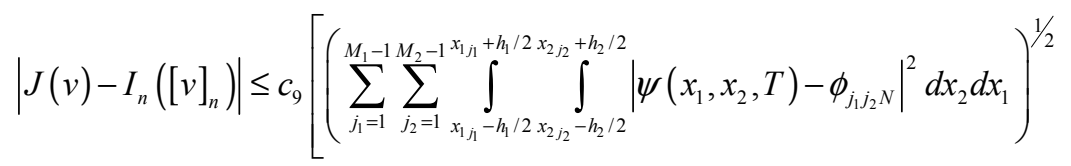

$$
\begin{aligned}
& \left.+\left(\sum_{j_{1}=1}^{M_{1}-1} \sum_{j_{2}=1}^{M_{2}-1} \int_{x_{1 / 1}-h_{1} / 2}^{x_{1}+x_{x_{2}}+h_{1} / 2} \int_{x_{2} / 2}^{x_{2} / h_{2} / 2}\left|y\left(x_{1}, x_{2}\right)-y_{j_{1} j_{2}}\right|^{2} d x_{2} d x_{1}\right)^{1 / 2}\right] \leq c_{10}\left[J_{1}+J_{2}\right]
\end{aligned}
$$

Here the number $c_{10}>0$ is independent $h_{1}, h_{2}$ and $\tau$.

Firstly let us evaluate the term $J_{1}$. To this, the following inequality can be written:

$$
\begin{aligned}
& J_{1}^{2}=\sum_{j_{1}=1}^{M_{1}-1} \sum_{j_{2}=1}^{M_{2}-1} \int_{x_{1 / 1}-h_{1} / 2}^{x_{1 / 1}+h_{2} / 2 x_{2}-h_{2} / 2} \int_{x_{2 / 2}+h_{2} / 2}\left|\psi\left(x_{1}, x_{2}, T\right)-\phi_{j_{1} j_{2} N}\right|^{2} d x_{2} d x_{1} \\
& \leq 2 \sum_{j_{1}=1}^{M_{1}-1} \sum_{j_{2}=1}^{M_{2}-1} \int_{x_{j_{1}}-h_{1} / 2}^{x_{1}+h_{j_{2}} / 2} \int_{x_{22}-h_{2} / 2}^{x_{2 / 2}+h_{2} / 2}\left|\psi\left(x_{1}, x_{2}, T\right)-\psi_{j_{1} j_{2} N}\right|^{2} d x_{2} d x_{1}+2 h_{1} h_{2} \sum_{j_{1}=1}^{M_{1}-1} \sum_{j_{2}=1}^{M_{2}-1}\left|\phi_{j_{1} j_{2} N}-\psi_{j_{1} j_{2} N}\right|^{2} \\
& =J_{11}+J_{12} \text {. }
\end{aligned}
$$

Using (34), we can write the following inequality:

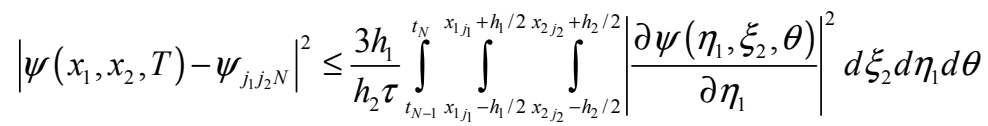

$$
\begin{aligned}
& +\left.\frac{3 h_{2}}{\tau} \int_{t_{N-1} x_{2 / 2}-h_{2} / 2}^{t_{N}} \int_{x_{2 / 2}+h_{2} / 2}^{x_{2}} \frac{\partial \psi\left(x_{1}, \eta_{2}, \theta\right)}{\partial \eta_{2}}\right|^{2} d \eta_{2} d \theta+3 \tau \int_{t_{N-1}}^{t_{\mathrm{N}}}\left|\frac{\partial \psi\left(x_{1}, x_{2}, \sigma\right)}{\partial \sigma}\right|^{2} d \sigma .
\end{aligned}
$$

Using the last inequality in the term $J_{11}$, we have:

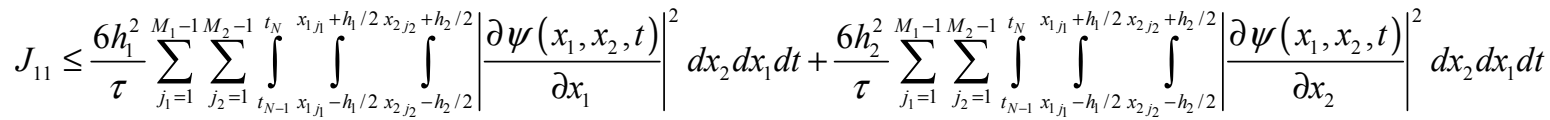

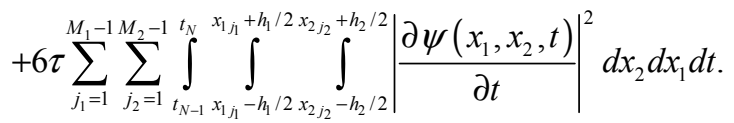

Considering adaptation conditions and (10) in last inequality, we get the following:

$$
J_{11} \leq c_{11}\left(h_{1}+h_{2}+\tau\right) .
$$

Here the number $c_{11}>0$ is independent $h_{1}, h_{2}$ and $\tau$.

Using $Z_{j_{1} j_{2} N}=\phi_{j_{1} j_{2} N}-\psi_{j_{1} j_{2} N}$ and (40), we have:

$$
J_{12} \leq c_{9}\left(\beta_{\tau h_{1} h_{2}}+\left\|Q_{n}(v)-[v]_{n}\right\|^{2}\right)
$$

Here the number $c_{9}>0$ is independent $h_{1}, h_{2}$ and $\tau$.

Now let us evaluate the term $J_{2}$. Considering the formula of $y_{j_{1} j_{2}}$, we can write the following:

$$
\begin{aligned}
& J_{2}^{2}=\sum_{j_{1}=1}^{M_{1}-1} \sum_{j_{2}=1}^{M_{2}-1} \int_{x_{1, j}-h_{1} / 2}^{x_{1, j}+h_{1} / 2} \int_{x_{2,2}-h_{2} / 2}^{x_{2, j}+h_{2} / 2}\left|y\left(x_{1}, x_{2}\right)-y_{j_{1} / j_{2}}\right|^{2} d x_{2} d x_{1}
\end{aligned}
$$

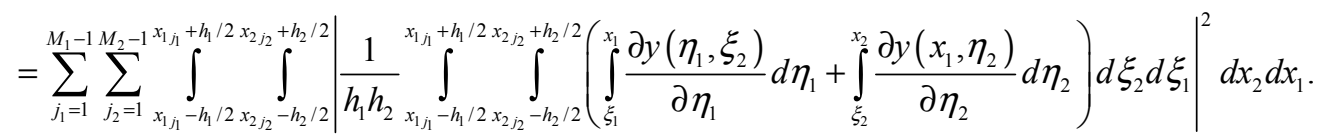

Applying Cauchy-Bunyakovsky inequality, we get following: 


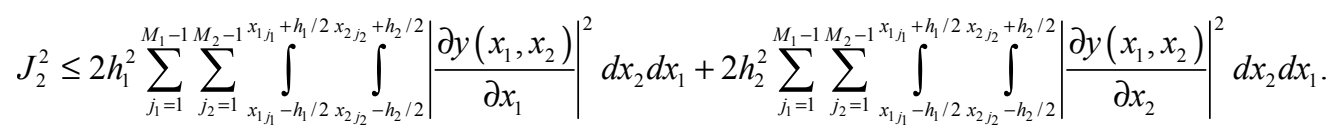

We easily have the following from the last inequality:

$$
J_{2}^{2} \leq c_{12}\left(h_{1}^{2}+h_{2}^{2}\right) .
$$

Here the number $c_{12}>0$ is independent $h_{1}$ and $h_{2}$. Using (44)-(46) in inequality (42), we get the following:

$\left|J(v)-I_{n}\left([v]_{n}\right)\right| \leq c_{10}\left[J_{1}+J_{2}\right] \leq c_{13}\left(\sqrt{\beta_{\tau h_{1} h_{2}}}+\left\|Q_{n}(v)-[v]_{n}\right\|\right)$.

Here the number $c_{13}>0$ is independent $h_{1}, h_{2}$ and $\tau$.

Lemma 1: Accept that the conditions of Theorem 4 are hold. Furthermore let the operator $Q_{n}(v)$ is defined by the formula (35). Then $Q_{n}(v) \in V_{n}$ for $\forall v \in V$ and the following estimate is valid:

$$
\left|J(v)-I_{n}\left(Q_{n}(v)\right)\right| \leq c_{8} \sqrt{\beta_{\tau h_{1} h_{2}}} .
$$

Lemma 2: Accept that the conditions of Theorem 4 are hold and let the operator $P_{n}$ is defined by the following formula:

$$
\begin{aligned}
& P_{n}\left([v]_{n}\right)=\tilde{v}(t)=\left(\tilde{v}_{1}(t), \tilde{v}_{2}(t)\right), \\
& \tilde{v}_{p}(t)=v_{p k}, t_{k-1} \leq t \leq t_{k}, k=\overline{1, N}, p=1,2 .
\end{aligned}
$$

Then $P_{n}\left([v]_{n}\right) \in V$ for $\forall[v]_{n} \in V_{n}$ and the following estimate is valid:

$$
\left|J\left(P_{n}\left([v]_{n}\right)\right)-I_{n}\left([v]_{n}\right)\right| \leq c_{8} \sqrt{\beta_{h_{1} h_{2} \tau}} .
$$

Theorem 5: Accept that the conditions of Lemma 1 and Lemma 2 are hold, $v^{*} \in V$ and $[v]_{n}^{*} \in V_{n}$ are solutions of problems (1)-(4) and (11)-(15), respectively. In other words let the equality satisfy the following:

$$
J\left(v^{*}\right)=J_{*}=\inf _{v \in V} J(v) \text { ve } I_{n^{*}}=I_{n}\left([v]_{n}^{*}\right)=\inf _{[v]_{n} \in V_{n}} I_{n}\left([v]_{n}\right) .
$$

Then the problem (11)-(15) is approximation of the optimal control problem (1)-(4). Namely the condition

$$
\lim _{n \rightarrow \infty} I_{n^{*}}=J_{*}
$$

is satisfied and the estimate

$$
\left|I_{n^{*}}-J_{*}\right| \leq c_{8} \sqrt{\beta_{h_{1} h_{2} \tau}}
$$

is valid for the convergence according to the functional.

Proof: Suppose that $v^{*} \in V$ is the arbitrary solution of the optimal control problem (1)-(4). $\quad Q_{n}\left(v^{*}\right) \in V_{n} \quad$ and $\left|I_{n}\left(Q_{n}\left(v^{*}\right)\right)-J\left(v^{*}\right)\right| \leq c_{8} \sqrt{\beta_{\tau h_{1} h_{2}}}, n=1,2, \ldots$ from Lemma 1.
Using this inequality, we have the following:

$$
I_{n^{*}} \leq I_{n}\left(Q_{n}\left(v^{*}\right)\right) \leq J\left(v^{*}\right)+c_{8} \sqrt{\beta_{\tau h_{1} h_{2}}}=J_{*}+c_{8} \sqrt{\beta_{\tau h_{1} h_{2}}}, n=1,2, \ldots
$$

Hence we write the following inequality:

$$
I_{n^{*}}-J_{*} \leq c_{8} \sqrt{\beta_{\tau h_{1} h_{2}}}, n=1,2, \ldots
$$

Let the control $[v]_{n}^{*} \in V_{n}$ is the arbitrary solution of the problem (11)-(15). $\quad P_{n}\left([v]_{n}^{*}\right) \in V$ from Lemma 2 and the following inequality is satisfied:

$$
\left|J\left(P_{n}\left([v]_{n}^{*}\right)\right)-I_{n}\left([v]_{n}^{*}\right)\right| \leq c_{8} \sqrt{\beta_{h_{1} h_{2} \tau}}, n=1,2, \ldots
$$

Hence the following inequality is valid:

$$
J_{*} \leq J\left(P_{n}\left([v]_{n}^{*}\right)\right) \leq I_{n}\left([v]_{n}^{*}\right)+c_{8} \sqrt{\beta_{h_{1} h_{2} \tau}}=I_{n^{*}}+c_{8} \sqrt{\beta_{h_{1} h_{2} \tau} \tau}, n=1,2, \ldots
$$

Using the last inequality, we can write the following inequality:

$$
I_{n^{*}}-J_{*} \geq-c_{8} \sqrt{\beta_{h_{1} h_{2} \tau}}, n=1,2, \ldots
$$

Hence we get the following from (53) and (54):

$$
\left|I_{n^{*}}-J_{*}\right| \leq c_{8} \sqrt{\beta_{h_{1} h_{2} \tau}}, n=1,2, \ldots
$$

Considering $\tau=\tau_{n}, h_{1}=h_{1 n}, h_{2}=h_{2 n}$ and $\lim _{n \rightarrow \infty} \tau_{n}=0$, $\lim _{n \rightarrow \infty} h_{1 n}=0, \lim _{n \rightarrow \infty} h_{2 n}=0$ we obtain $\lim _{n \rightarrow \infty} \beta_{h_{1 n} h_{2 n} \tau_{n}}=0$ for $\beta_{h_{1} h_{2} \tau}$. Using these in the estimate (52), we have $\lim _{n \rightarrow \infty} I_{n^{*}}=J_{*}$ for $n \rightarrow \infty$.

\section{References}

[1] H. Yetişkin, M. Subaşı, "On the optimal control problem for Schrödinger equation with complex potential," Applied Mathematics and Computation, 216, 1896-1902, 2010.

[2] B. Yıldız, O. Kılıçoğlu, G. Yagubov, "Optimal control problem for non stationary Schrödinger equation,” Numerical Methods for Partial Differential Equations, 25, 1195-1203, 2009.

[3] K. Beauchard, C. Laurent, "Local controllability of 1D linear and nonlinear Schrödinger equations with bilinear control," Journal de Mathematiques Pures et Appliquees, 94 (5), 520-554, 2010.

[4] B. Yıldız, M. Subaşı, "On the optimal control problem for linear Schrödinger equation. Applied Mathematics and Computation," 121, 373-381, 2001. 
[5] L. Baudouin, O. Kavian, J. P. Puel, "Regularity for a Schrödinger equation with singuler potentials and application to bilinear optimal control," Journal Differential Equations, 216, 188-222, 2005.

[6] G. Ya. Yagubov, M. A. Musayeva, "Finite-difference method solution of variation formulation of an inverse problem for nonlinear Schrodinger equation," Izv. AN Azerb.-Ser. Physictex. matem. nauk, vol.16, No 1-2,46-51, 1995.

[7] G. D. Smith,"Numerical Solution of Partial Differential Equations," Oxford University Press, 1985.
[8] J. V. Thomas, "Numerical Partial Differential Equations," Springer- Verlag, 1995.

[9] O. A. Ladyzhenskaya, V. A. Solonnikov, N. N. Uraltseva, "Linear and Quasi-Linear Equations of Parabolic Type, Translations of Mathematical Monographs," American Mathematical Society, Rhode Island, 1968.

[10] F. P. Vasilyev, "Numerical Methods for Extremal Problems," Nauka, Moskow, 1981. 